\title{
Tissue Spectroscopy and Optical Clearing of Colorectal Mucosa in the Pursuit of New Cancer Diagnostic Approaches
}

\author{
Luís Fernandes ${ }^{1}$, Hugo Silva ${ }^{1,2}$, Inês Martins ${ }^{1,2}$, Sónia Carvalho ${ }^{3,4}$, Isa Carneiro ${ }^{3}$, Rui Henrique ${ }^{3,5}$, \\ Valery V. Tuchin ${ }^{6,7,8}$, and Luís M. Oliveira ${ }^{1,2^{*}}$ \\ ${ }^{1}$ Physics Department, School of Engineering, Polytechnic Institute of Porto, \\ Rua Dr. António Bernardino de Almeida 431, 4249-015 Porto, Portugal \\ ${ }^{2}$ Center of Innovation in Engineering and Industrial Technology, ISEP, Rua Dr. António Bernardino de Almeida 431, \\ 4249-015 Porto, Portugal \\ ${ }^{3}$ Department of Pathology and Cancer Biology and Epigenetics Group-Research Center, \\ Portuguese Oncology Institute of Porto, 4200-072 Porto, Portugal \\ ${ }^{4}$ Department of Pathology, Santa Luzia Hospital, ULSAM, 4904-858 Viana do Castelo, Portugal \\ ${ }^{5}$ Department of Pathology and Molecular Immunology, Institute of Biomedical Sciences Abel Salazar-University of \\ Porto (ICBAS-UP), 4050-313 Porto, Portugal \\ ${ }^{6}$ Science Medical Center, Saratov State University, 83 Astrakhanskaya str., Saratov 410012, Russian Federation \\ ${ }^{7}$ Laboratory of Laser Molecular Imaging and Machine Learning, National Research Tomsk State University, \\ 36 Lenin's av., Tomsk 634050, Russian Federation \\ ${ }^{8}$ Laboratory of Laser Diagnostics of Technical and Living Systems, Precision Mechanics and Control Institute of the \\ Russian Academy of Sciences, 24 Rabochaya str., Saratov 410028, Russian Federation
}

*e-mail: Imo@isep.ipp.pt

\begin{abstract}
In this paper we present three studies that demonstrate the applicability of spectroscopy methods and optical clearing treatments in pathology identification and monitoring. In the first study, by obtaining the absorption spectra of human healthy and pathological (adenocarcinoma) colorectal mucosa tissues, it was possible to identify a higher content of a pigment in the diseased tissues. This study also shows that machine learning methods can be used to reach the same differentiated results in vivo through diffuse reflectance spectroscopy. In the second study, the combination of collimated transmittance spectroscopy with optical clearing treatments allowed to obtain the diffusion coefficients of glucose in healthy and pathological colorectal mucosa as: $D_{\text {glucose }}=5.8 \times 10^{-7} \mathrm{~cm}^{2} / \mathrm{s}$ and $D_{\text {glucose }}=4.4 \times 10^{-7} \mathrm{~cm}^{2} / \mathrm{s}$, respectively. This study also demonstrated that the diseased tissues contains about $5 \%$ more mobile water than the healthy tissues. The third study was performed to evaluate the protein dissociation mechanism of optical clearing. By treating both healthy and pathological colorectal mucosa tissues with 93\%-glycerol, a protein dissociation rate of about 3 times higher was obtained for the pathological mucosa. All the discriminating parameters that result from these studies can be obtained in the in vivo situation through diffuse reflectance spectroscopy and further studies to evaluate their values in different stages of cancer progression are of great importance to develop disease monitoring protocols. (C) 2021 Journal of Biomedical Photonics \& Engineering.
\end{abstract}

Keywords: colorectal cancer; tissue optical clearing; optical clearing mechanisms; cancer detection; enhanced tissue spectroscopy.

Paper \#3447 received 31 Jul 2021; revised manuscript received 24 Aug 2021; accepted for publication 27 Aug 2021; published online 8 Sep 2021. doi: 10.18287/JBPE21.07.040302. 


\section{Introduction}

Current diagnostic of oncologic malignancies relies on imaging methods that use ionizing radiation [1]. Such procedures present a great number of disadvantages, since ionizing radiation can induce, among others, DNA double-strand breaking $[2,3]$ and cell death in surrounding healthy tissues $[4,5]$. To avoid such side effects of ionizing radiation, biophotonics suggests alternative optical methods that, by the harmless use of light, can perform reliable and noninvasive diagnostic procedures. To develop such methods, the cancer-related biophotonics research has been growing in the past 30 years, with an increasing number of publications, as we have found in Pubmed, Google Scholar and ScienceDirect, when searching with the term "Optical cancer detection".

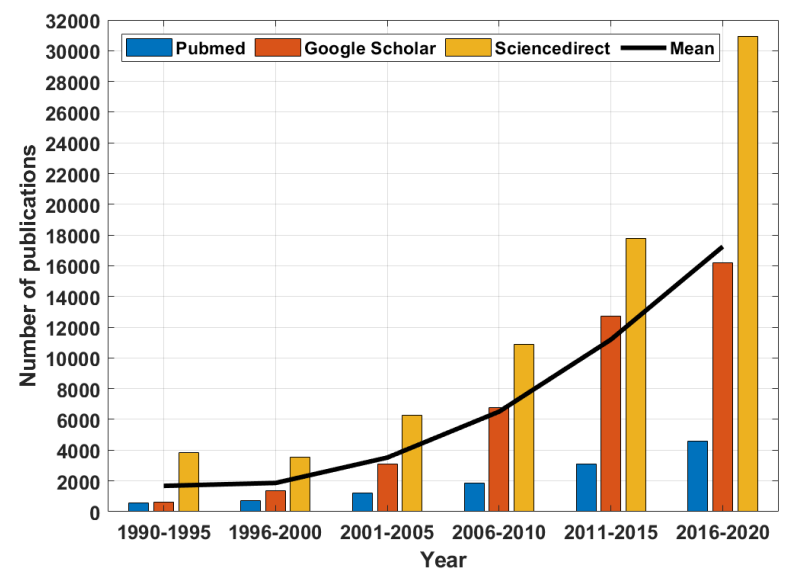

Fig. 1 Number of publications related to optical detection of cancer between 1990 to 2020 .

The optical methods used in the studies of most of these publications are basically included in two groups: spectroscopy methods, which include optical, Raman, light scattering, near infrared and fluorescence, and imaging methods, which include optical coherence tomography (OCT), fluorescence microscopy, laser scanning microscopy and confocal microscopy.

Considering the development of any type of cancer, space- and time-dependent variations, such as abnormal cell growth, occur in tissues. When the later stages of cancer progression are reached, malignant tumors develop and cancer cell metastasis occur, creating the major cause of morbidity and mortality in a high number of patients [1,6-13]. Nowadays, the classification of the cancer types is made according to the associated fatality degree or according to the anatomical area where they occur [14-17]. The detection, explanation and control of cancer progression are difficult for clinicians, since it can occur in different anatomical areas of the body without any apparent reason and cancer cells can spread to other areas. An additional fact is that any therapeutic procedure to eliminate cancer becomes ineffective when the diagnosis is established at a later stage of progression, which is the most common case $[1,18]$.
To overcome such difficulties, biophotonics methods can be used to detect cancer, even at early stages of development, and eventually aid in creating a delay in its progression.

In the case of early stage of cancer development, if the polyps or tumors arise at the outermost tissue layers, noninvasive or minimally invasive imaging or spectroscopy methods may be used directly or through endoscopy to perform a diagnosis. In such clinical exams, due to the early stage of cancer development, the clinician may have doubts to establish a reliable diagnosis, but through the precise optical identification of cancer biomarkers, a correct diagnosis can be established and treatment measures can be adopted to extend life expectancy for the patient. One such example was recently reported by our group with the identification of discriminated content of a lipofuscin-type pigment in healthy and pathological (colorectal cancer adenocarcinoma) tissues of the human colorectal mucosa [19]. Although such study was made with ex vivo tissues, a similar evaluation of the discriminated pigment content can be made in vivo, as recently reported by Fernandes et al. [20]. In this study, diffuse reflectance measurements that mimic the noninvasive in vivo situation were used as input to develop machine learning (ML) algorithms to calculate the absorption coefficient spectra of healthy and pathological colorectal mucosa. The results obtained in this study were similar to the ones previously obtained in our study [19], with invasive measurements performed from ex vivo tissue samples collected from surgical specimens, and allowed to discriminate the lipofuscin-type pigment as before. Such results obtained by the ML approach open the way for in vivo evaluation of the pigment content in pathological tissues as a function of cancer progression to establish a diagnosis and identify the stage of cancer evolution.

For the case where such cancer biomarkers arise at the inner tissue layers, the direct application of imaging or spectroscopic procedures is not efficient, since tissues present high scattering properties, which limits the light probing depth [21]. Considering in particular the spectroscopy approach, biological tissues have various components, with individual absorption bands, which most of the times overlap one another, especially in the ultraviolet (UV) spectral range [22]. The discrimination of such useful spectral signatures for the establishment of a precise diagnosis may also be camouflaged by the strong and multiple light scattering that occur in biological tissues [23].

To overcome, such high and multiple scattering and reach deeper tissue layers to perform a reliable diagnosis, the application of optical clearing (OC) treatments to the tissues may be adopted [24]. Such method has been intuitively applied by Man since ancient times [25], but the first scientific evidence of the induced transparency increase in biological tissues was reported more than a century ago by a German anatomist, named Werner Spalteholz $[26,27]$. No practical use of this method existed by that time, but with the development of new 
optical instruments in the second half of the XX century, such as the laser, optical fibers or light-emitting-diodes (LEDs) of different colors, with efficient emission, a new era of light application in biology and medicine has arisen [21]. Consequently, new interest to study the light propagation in biological tissues, the light beam interaction with biological components in tissues and also the light scattering reduction that occurs as a consequence of the OC treatments has emerged [28].

The study of OC treatments has been intensively performed in the last 20 years, allowing to obtain good results in many biological applications, both on ex vivo and in vivo tissues [21,24]. New approaches for cancer detection and the possibility of early stage detection and cancer progress monitoring can be provided by the combination of $\mathrm{OC}$ treatments with imaging or spectroscopic methods [18, 21].

The main purpose of the OC treatments is to increase the transparency of biological tissues through the reduction of light scattering inside. Such transient effect allows to reach deeper light probing depths through the partial replacement of tissue water by a harmless agent, designated as optical clearing agent (OCA) [21]. These OCAs have a higher refractive index (RI) than water, better matched to the RI of the other tissue components, which are commonly designated as tissue scatterers [21, 28, 29]. Due to heterogeneous composition of biological tissues, the various biological components such as protein fibers and globules, lipid droplets, phospholipid membranes, cells and their organelles, like mitochondria and nucleus, are surrounded by the tissue fluids, e.g., the interstitial fluid (ISF) or cell cytoplasm [30]. These tissue fluids contain a small amount of salts, minerals and organic compounds, which are dissolved in the main fluid component water [24, 31, 32]. Water in tissue fluids can reach values above 95\% [28], which decreases the RI of fluids strongly. Part of tissue water is also bound to tissue scatterers to keep them hydrated, but such amount of water is small, meaning that the RI of tissue scatterers is significantly higher than the RI of tissue fluids. As an example, the RI values of dry proteins in skeletal muscle and skin melanin at $589.6 \mathrm{~nm}$ are 1.584 and $1.47[21,24,32,33]$, respectively, while the RI of the ISF in most tissues is of the order of 1.35-1.37 [24]. The strong and multiple light scattering that occurs in biological tissues is originated due to this RI mismatch between the ISF and the tissue scatterers [21].

As a result of the intense research in this field, various OCAs have given proof of efficiency in the creation of temporary and reversible transparency in different soft and hard tissues and in blood [21, 24]. Considering safety issues, the great majority of these OCAs have not induced side effects in tissues [21, 24], but some exceptions have been reported for some tissues under treatment with highly concentrated solutions of glycerol, dimethyl sulfoxide (DMSO) and propylene glycol (PG) $[21,34,35]$. The OCAs that are known today can have different nature, but they are in general classified in three main groups: sugars, alcohols and electrolyte solutions [21]. In the group of sugars, we find maltose, fructose and glucose, while in the group of alcohols we can find glycerol, sorbitol or PG. Considering the group of electrolyte solutions, these are commercial products to be used as contrast agents in medical imaging procedures, and some examples are Trazograph ${ }^{\mathrm{TM}}$, Hypaque $^{\mathrm{TM}}$ and Gadolinium ${ }^{\mathrm{TM}}[21,23,24,36]$. Tests with these and other OCAs have been made in various animal and human tissues, both in vivo and ex vivo and the created transparency varies both in magnitude and duration from tissue to tissue [21]. Such transparency created by these OCAs in biological tissues is obtained by the cooperation of three main mechanisms, designated as: tissue dehydration, RI matching and protein dissociation [21].

To explain in detail how these mechanisms work and lead to a reduction in light scattering in tissues, we will consider a simple ex vivo tissue sample, which was prepared to have a slab-form with $0.5 \mathrm{~mm}$ thickness and a diameter of $\sim 1 \mathrm{~cm}$. This sample is submitted to collimated transmittance $\left(T_{\mathrm{c}}\right)$ measurements, both in its natural state and during $\mathrm{OC}$ treatment to evaluate its kinetics. The Beer-Bouguer-Lambert law states that $T_{c}$ is a function of the attenuation coefficient, $\mu_{t}$, and sample thickness, $d[23]$ :

$$
T_{c}(\lambda) \approx e^{-\mu_{t}(\lambda) \times d}
$$

where $\lambda$ represents the wavelength and $\mu_{t}$ is the sum of the absorption coefficient, $\mu_{a}$, with the scattering coefficient, $\mu_{s}$.

Considering as a reference the $T_{c}$ spectrum for a selected wavelength range that was measured from the natural sample, when the treatment begins by immersing the tissue sample in a solution that contains an OCA, an increase in $T_{c}$ will be seen as a result of the $\mathrm{OC}$ mechanisms. In the first few minutes of treatment, the osmotic pressure of the OCA in the solution induces the water flux out from the tissue. This is the dehydration mechanism [21]. Depending on the bounding strength, there are four stages of water in biological tissues that have been reported [37,38]: strongly bound, tightly bound, weakly bound and free water. The combination of weakly bound with free water is commonly designated as mobile water, since both can move to the outside of the tissue when stimulated by the osmotic pressure of the OCA used in the treatment [21]. The osmotic pressure created by the OCA is not strong enough to break the connections between the strongly and tightly bound water and the other biological components. Such water maintains the hydration of those biological components in the tissues and provide nutrients to the cells, but it can also be converted into mobile water and flow out if the osmotic pressure of the OCA is maintained for several hours or days [21]. As a result of the fast loss of mobile water in the first few minutes of treatment, sample thickness decreases, leading to the close approximation of the tissue scatterers [21, 29, 39]. A more compact and better organized packing of tissue scatterers is created 
inside the sample [23, 24, 40, 41]. Mechanical compression or stretching may also induce such internal variations, since mechanical forces also remove water from the compressed or stretched area of the tissue $[21,24]$. An increase in $\mu_{s}$ and in $\mu a$ should be expected with the creation of a denser scatterer distribution inside the tissue, but due to a better scatterer ordering inside and to the decreased sample thickness, an increase in transparency occurs [23, 33, 39].

As the mobile water flows out, the diffusion of the OCA molecules into the interstitial locations is facilitated. Since these molecules have a higher RI than the water, the OCA positioning in the interstitial locations, and in particular close to the tissue scatterers, provides the RI matching mechanism $[21,39]$. As the OCA molecules start their diffusion into the tissue, they begin their interaction with the outermost tissue layers, but with the continuous flux of the water molecules going out, the OCA molecules flow into deeper locations and eventually fill all the interstitial areas of the tissue. The OCA diffusion into the tissues takes several minutes to occur, as demonstrated by various studies [40, 42, 43]. Since both fluxes initiate at the beginning of the treatment, during the first few minutes of treatment both the water flux going out and the OCA flux going in occur simultaneously. This means that methods for flux discrimination and characterization are necessary.

The replacement of interstitial water by the OCA molecules results in an increase of the mean RI in the ISF, approaching it to the RI of tissue scatterers - this is the RI matching mechanism, which is provided by the OCA inclusion in the interstitial locations [40-42]. This mechanism decreases the light scattering inside the tissue, leading also to the increase of tissue transparency [43]. Since the OCA molecules can be washed out by the water that flows from adjacent tissues in vivo or by immersing the ex vivo tissue sample in saline, both the tissue dehydration and the RI matching mechanisms are reversible [39]. One OC study demonstrated that the increased transparency created in skin by these two mechanisms has provided better contrast images from deeper tissue layers [44]. Another study performed with human ex vivo healthy and diseased colorectal tissues to discriminate and characterize the OC mechanisms demonstrated a 5\% higher content of mobile water in the pathological tissues [43, 45]. Similar results for a higher mobile water content in pathological tissues were observed in a study performed with tissues from healthy and diabetic mouse [46].

A third OC mechanism that also leads to the increase of tissue transparency is designated as protein dissociation. This third mechanism of OC was first observed in 2003, in a study where multiphoton microscopy was used to generate second harmonic generation (SHG) images from ex vivo skin samples under treatment with glycerol solutions [47-49]. In this study, and as a result of the applied treatments, it was observed that collagen I dissociation occurs in the extracellular skin matrix due to the action of glycerol. Since the reassembly of skin matrix collagen I was observed after tissue rehydration in saline in a later study by the same group, the reversibility of this third OC mechanism was demonstrated $[47,50]$. The protein dissociation mechanism and its reversibility after washing out the OCA from the tissues have also been demonstrated with other OCAs, such as ethylene glycol (EG) and sorbitol [47, 49]. These studies showed that the clearing potential of an OCA is related to its protein dissociation capability, proving that the protein dissociation is also an OC mechanism [47-50]. More recently, other studies have showed that this mechanism also occurs in other tissues such as colorectal muscle [25], or colorectal mucosa [29]. In the first of these studies it was demonstrated that the protein dissociation increases in magnitude with the osmolarity of glycerol in the treating solution [25]. In the later study [29], Carneiro et al. demonstrated that the protein dissociation rate can be discriminated in healthy and pathological colorectal mucosa for the same high glycerol osmolarity in the treating solution. Such result of discriminated protein dissociation rates in healthy and cancer tissues is to be expected due to the higher protein contents that cancer tissues are known to have [51].

In the past 5 years, the cooperation between our groups, located in Porto (Portugal) and in Saratov (Russian Federation), has originated various results with studies using ex vivo healthy and diseased tissues, that can be used to develop future noninvasive optical methods for cancer and diabetes detection and monitoring. The purpose of this paper is to review those discriminating results obtained for the case of human colorectal cancer. Section 2 describes the methodologies used in these studies and the obtained results are presented in Section 3.

\section{Materials and methods}

The studies that originated the results presented in Section 3 were all conducted with ex vivo tissue samples. The following Subsections describe the tissue collection and preparation, the spectroscopy and thickness measurements and the calculation procedures used in the different studies to obtain the results that discriminate colorectal cancer.

\subsection{Tissue samples}

Human colorectal surgical resections were collected from adult patients under treatment at the Oncology Institute of Porto (Portugal). These patients have previously signed a written consent, allowing the use of surgical specimens for diagnostic and research purposes.

The healthy and pathological areas of the surgical resections were separated and the tissue samples were prepared using only the mucosa layer of the colorectal wall. Such samples were prepared using a cryostat (Leica, model CM $1850 \mathrm{UV}$ ) to have an approximately circular-slab form with a diameter of $\sim 1 \mathrm{~cm}$ and a uniform thickness of $500 \mu \mathrm{m}$. The number of healthy and pathological samples used in the various studies are indicated in the following subsections. 


\subsection{Experimental studies}

To perform the various studies, it was necessary to conduct both spectroscopy and thickness measurements. In the first study to estimate the differentiated accumulation of a pigment in healthy and pathological mucosa, only spectroscopy measurements were made. To conduct this study, 10 healthy and 10 pathological mucosa samples were submitted to total transmittance $\left(T_{t}\right)$, total reflectance $\left(R_{t}\right)$ and $T_{c}$ measurements [19]. An additional set of 10 healthy and 10 pathological mucosa samples was later used to measure the diffuse reflectance $\left(R_{d}\right)$ spectra, with the objective of estimating the $\mu_{a}$ spectra for both tissues using ML algorithms.

In the second study to obtain the differentiated mobile water content in healthy and pathological mucosa, $T_{c}$ measurements were made from samples under treatment with aqueous solutions containing different glucose concentrations: $10 \%, 15 \%, 20 \%, 25 \%, 30 \%, 35 \%, 40 \%$, $45 \%, 50 \%$ and $54 \%$. Three studies with each type of tissue were made for each treatment with the previous solutions to obtain statistical data. To calculate the diffusion coefficients of water and glucose in healthy and pathological mucosa, additional thickness measurements were made during treatment with some of the above solutions $[43,45]$. A total of 6 healthy and 6 pathological tissue samples were used in the thickness measurements.

In a previous study [25], we observed that OC treatments with strong OCA osmolarity induce the creation of a high magnitude UV window between 200 and $260 \mathrm{~nm}$. Due to this knowledge, we planned a new study to evaluate the protein dissociation rate in healthy and pathological colorectal mucosa. In this third study, 5 healthy and 5 pathological tissue samples were submitted to $T_{c}$ spectral measurements during treatment with $93 \%$ glycerol [29].

The following sub-sections present the experimental setups and describe the measuring procedures adopted in the three studies.

\subsubsection{Total transmittance}

Fig. 2 presents the experimental setup used to measure the $T_{t}$ spectra from the tissue samples.

In these measurements, the sample was irradiated with a high-power pulsed broad-band xenon lamp through an optical fiber cable and collimating lens. The incident beam had a diameter of $6 \mathrm{~mm}$, which is limited by the lens aperture (indicated in Fig. 2 as the pinhole). The light that is transmitted by the tissue sample enters the integrating sphere to be reflected several times on its inside wall (integration) before being delivered to the spectrometer through a second optical fiber cable. All these equipments were acquired from Avantes ${ }^{\mathrm{TM}}$ (Netherlands). All spectra measured with this setup from 10 healthy and 10 pathological mucosa samples were acquired between 200 and $1000 \mathrm{~nm}$ to be used in the first study that is presented in Section 3.

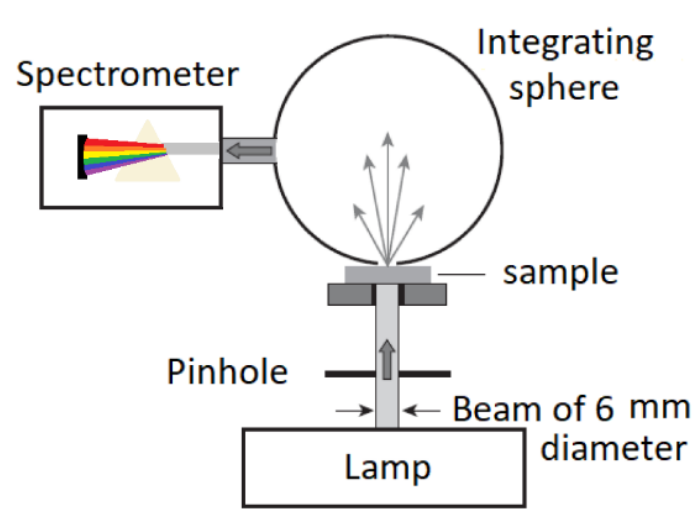

Fig. 2 Total transmittance $T_{t}$ measuring setup with integrating sphere.

\subsubsection{Total reflectance}

The setup used to measure the $R_{t}$ spectra from samples is presented in Fig. 3.

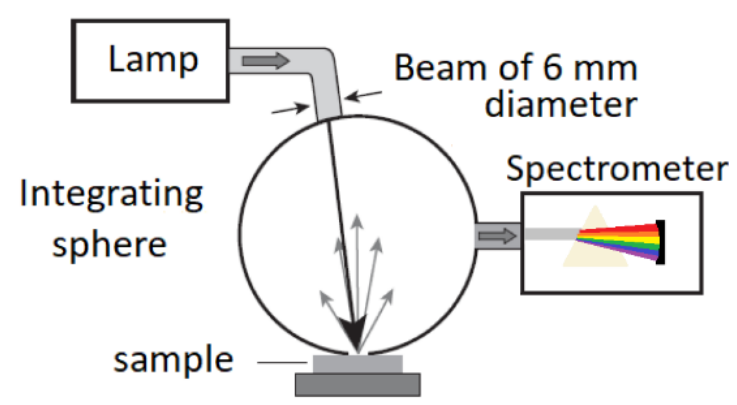

Fig. 3 Total reflectance $R_{t}$ measuring setup with integrating sphere.

In a similar manner to what was described for the measurements with the $T_{t}$ setup, a high-power pulsed broad-band xenon lamp is used to irradiate the tissue sample through an optical fiber cable and collimating lens. Such sample irradiation is made at $8^{\circ}$ with the vertical axis of the integrating sphere. The beam is reflected by the sample and then is submitted to the integration process inside the sphere, meaning that it undergoes multiple reflections before being delivered to the spectrometer through an optical fiber cable. Considering these measurements for the first study that is presented in Section 3, 10 spectra were acquired from healthy tissue samples and other 10 from the pathological samples. All spectra were acquired between 200 and $1000 \mathrm{~nm}$.

\subsubsection{Diffuse reflectance}

Fig. 4 presents the experimental setup that was used to measure the $R_{d}$ spectra that were used to estimate $\mu_{a}(\lambda)$ for both mucosa tissues with the ML algorithms.

In these measurements, a broad-band beam from a deuterium-halogen lamp (Avantes ${ }^{\mathrm{TM}}$, Netherlands) irradiates the sample through an optical fiber that is placed inside the $R_{d}$ optical fiber sensor (ArtPhotonics ${ }^{\mathrm{TM}}$, Germany). The tip of this sensor is maintained $2 \mathrm{~mm}$ 
above the surface of the tissue sample. The diffusively reflected light is collected by other 6 optical fibers in the sensor, which deliver such reflected light to the spectrometer. $R_{d}$ spectra were acquired from 10 healthy and from 10 pathological mucosa tissue samples, between 200 and $1000 \mathrm{~nm}$.

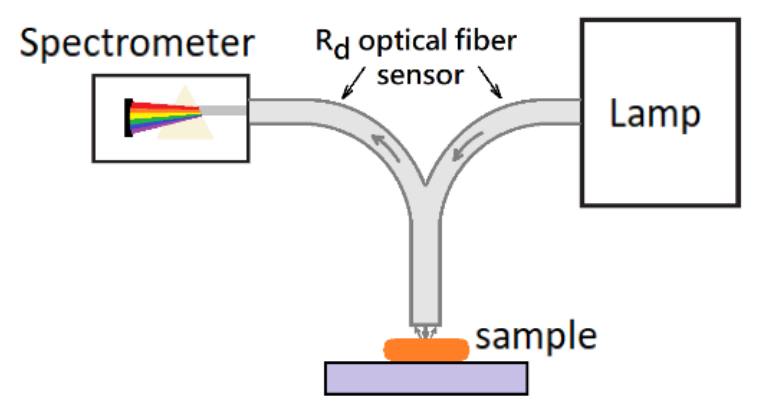

Fig. 4 Diffuse reflectance $R_{d}$ measuring setup.

\subsubsection{Collimated transmittance}

The $T_{c}$ setup that is presented in Fig. 5 was used to acquire spectra from tissues in all studies.

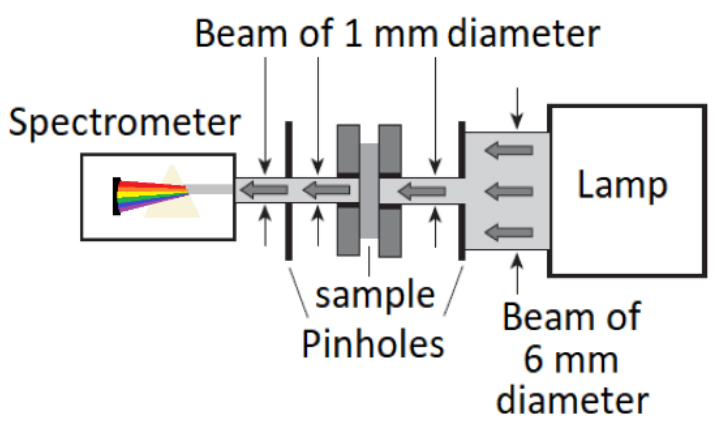

Fig. 5 Collimated transmittance $T_{c}$ measuring setup.

In all measurements made with this setup, a beam from a broad-band deuterium-halogen lamp (the same that was used in the $R_{d}$ measurements) was delivered to the right side of the sample through an optical fiber cable and collimating lens. Having a beam diameter of $6 \mathrm{~mm}$ as it exits the lens, the beam crosses a pinhole that reduces its diameter to $1 \mathrm{~mm}$, before irradiating the tissue sample. A second pinhole and another collimating lens placed on the left side of the sample are the collecting optics used to deliver the transmitted beam to an optical fiber cable that is connected to the spectrometer. All spectra acquired with this setup are registered between 200 and $1000 \mathrm{~nm}$. The space between the two pinholes is inside a dark cuvette that prevents the entrance of ambient light and contains the OC solution surrounding the tissue sample when measurements are to be made during the treatments. The sample is fixed at the center of this cuvette by a mechanical system, which is represented by the four dark-grey rectangles on both sides of the sample in Fig. 5. The beam entrance and exit in the cuvette is made through two special glasses that have flat transmittance (above 90\%) through the entire spectral range of interest (200 to $1000 \mathrm{~nm}$ ). For measurements regarding the first study, 10 spectra were acquired from the same number of samples. For the spectral measurements performed in the second study, three independent studies were performed with the same number of tissue samples for the treatments with each glucose concentration. In the case of the third study, 5 healthy and 5 pathological tissue samples were submitted to measurements with the setup in Fig. 5 during treatment with $93 \%$-glycerol. In all studies, when measuring the native $T_{c}$ spectra of the tissue samples, the setup is used as represented in Fig. 5. For the measurements during treatments in the second and third studies, the cuvette is filled with the OCA solution to immerse the sample before starting the measurements. In these measurements, a $T_{c}$ spectrum is acquired at each $5 \mathrm{~s}$ during $30 \mathrm{~min}$.

\subsubsection{Thickness measurements}

In the second study, which was made to obtain the mobile water content in the healthy and pathological colorectal mucosa tissues and the diffusion properties of glucose in these tissues, thickness measurements were necessary during treatment of the tissues with some solutions. Those measurements were made by placing the tissue sample inside two microscope glasses as represented in Fig. 6.

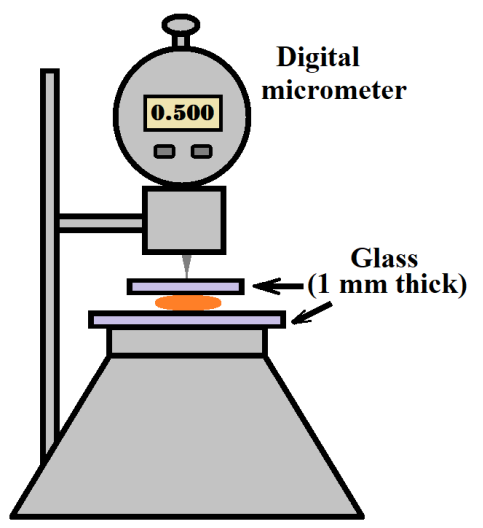

Fig. 6 Setup for measuring of tissue sample thickness.

Each of the two glasses had a fixed thickness of $1 \mathrm{~mm}$, but to avoid excessive force over the sample and unwanted sample compression during measurements, the one on top had a smaller area $(1.5 \times 1.5 \mathrm{~cm})$ than the one at the bottom $(1.5 \times 7.5 \mathrm{~cm})[48]$.

The digital micrometer represented in Fig. 6 had a precision of $0.001 \mathrm{~mm}$ and was used to measure the global thickness of the tissue sample between the two glasses. To perform a thickness kinetics study the micrometer was adjusted to have a reading of $2.500 \mathrm{~mm}$ at the beginning. Then, a significant amount of the treating solution was injected in-between the two glasses to immerse the entire sample and initiate the treatment. The micrometer was adjusted smoothly to measure new thickness values at each $15 \mathrm{sec}$ in the first $2 \mathrm{~min}$ and at each min after that. Considering the healthy mucosa tissues, thickness measurements were performed during 
treatment with the $40 \%$ - and 54\%-glucose solutions. For the case of pathological mucosa tissues, those measurements were performed during treatment with the $35 \%$ - and 54\%-glucose solutions. Three sets of thickness measurements were made from independent mucosa samples under treatment with each of these solutions to obtain statistical thickness kinetics variations $[43,45]$.

\subsection{Calculations}

After terminating the experimental measurements for each study, some calculations needed to be performed. Considering the first study to evaluate the differentiated pigment content in the healthy and pathological mucosa tissues, the first calculation consisted on obtaining the spectrum of $\mu_{a}$ from the $T_{t}$ and $R_{t}$ spectra. Such calculation was made 10 times for the healthy and 10 times for the pathological mucosa, using Eq. (2) [19]:

$$
\mu_{a}(\lambda)=\frac{1-\frac{T_{t}(\lambda)+R_{t}(\lambda)}{100}}{0.05} .
$$

In Eq. (2), the number 100 is a normalization factor for the spectral measurements and the number 0.05 represents the sample thickness in $\mathrm{cm}$. Once the mean $\mu_{a}(\lambda)$ was calculated for each tissue, we observed that the ratios between the hemoglobin peaks at 415 and $550 \mathrm{~nm}$ and the minimal value of $\mu_{a}$ in the near infrared (NIR) were significantly low when compared to others previously published for similar mucosal tissues [52]. This difference between our data and the one that was previously published means that some pigment is creating an additional absorption in the entire spectral range in our tissues, and consequently camouflaging their real blood content. By obtaining information from the patient records at the Portuguese Oncology Institute of Porto, we found that due to a natural product that was administered to the patients in the two days before the colorectal surgery, some lipofuscin-type pigment was detected in the complementary histological analysis. Obtaining the absorption spectrum of lipofuscin $\left(\mu_{\text {a-pigment }}\right)$ from literature, as measured from other tissues [53], we reconstructed it as in Eq. (3) [19], to be subtracted to the $\mu_{a}$ of the healthy colorectal mucosa and obtain appropriate ratios for the absorption bands of hemoglobin in this tissue.

$$
\mu_{a-p i g m e n t}(\lambda)=A \times\left[5.2+e^{(3.524-0.01087 \times \lambda)}\right] .
$$

In Eq. (3) $\lambda$ is to be used in nm to obtain the values of $\mu_{\text {a-pigment }}$ in $\mathrm{cm}^{-1}$. $A$ represents the percentage of the pigment in the tissue, which for the case of the healthy mucosa assumes the value 1 (100\% content). For the case of the pathological mucosa a similar calculation could be made, but considering $A$ in Eq. (3) as 1.1 ( $110 \%$ content). By subtracting the pigment content from the absorption spectra of both tissues, we obtained proper ratios for the hemoglobin bands in the healthy tissue and a higher blood content in the pathological mucosa as it was expected [19]. The results of this study are presented in Section 3.

Regarding the second study to calculate the mobile water content and the diffusion coefficients of glucose in healthy and pathological mucosa, we used both thickness and $T_{c}$ kinetics data obtained from the various treatments with glucose solutions. Since both the healthy and the pathological mucosa presented an almost linear increasing behavior of $T_{c}$ with wavelength between 600 and $800 \mathrm{~nm}$ [45], we selected individual wavelengths within this range to calculate the $T_{c}$ kinetics for each treatment. To calculate these kinetics, we used mean spectra from three studies for each particular treatment $[43,45]$. Since the $T_{c}$ kinetic measurements are sensitive to the variations induced by glucose during the treatments, we adjusted such kinetics data set obtained for each individual wavelength with a curve as described by Eq. (4) [45]:

$$
T_{c}(\lambda, t)=\frac{C_{a}(t)}{C_{a 0}} \cong\left[1-\exp \left(-\frac{t}{\tau}\right)\right],
$$

with

$$
\tau=\frac{d^{2}}{\pi^{2} D_{O C A / \text { water }}},
$$

where $C_{a}(t)$ is the OCA concentration inside the tissue at a particular time of treatment $t, C_{a}$ is the initial OCA concentration in the treating solution, $\tau$ represents the characteristic diffusion time of the effective global flux between the treating solution and the tissue, which is related through Eq. (5) to the characteristic diffusion coefficient of that effective global flux (DocA/water) and $d$ represents the sample thickness at a time of treatment that corresponds to $\tau$ [45]. Our objective in this study was to discriminate the characteristic diffusion properties ( $\tau$ and $D$ ) for glucose and water in both mucosa tissues. Those properties can only be retrieved using data from treatments where a unique glucose or water flux occurs between the treating solution and the tissue. The unique water flux is obtained from a treatment with a high concentration of the OCA in the treating solution. In our case we were using glucose and the highest glucose concentration possible in aqueous solution is 54\% [21]. The unique glucose flux is obtained when the water in the treating solution is in equilibrium with the mobile water in the tissue, but we did not know this value for the healthy and pathological mucosa tissues before the study was made. This way, we needed to perform various studies with treating solutions containing different glucose concentrations. From each treatment with a particular solution, we obtained various $\tau$ values in the fittings of the $T_{c}$ kinetics data for each selected wavelength with Eq. (4). By representing the mean $\tau$ values obtained for each treatment as a function of the glucose concentration in the treating solution for each 
tissue, we obtained a dependence that provided a maximum and a minimum $\tau$ value. For any tissue, the minimum $\tau$ value represents the characteristic diffusion time of water and the maximum $\tau$ value represents the characteristic diffusion time of glucose in that tissue. These $\tau$ values that were retrieved from those graphical representations were used in Eq. (5) to calculate the diffusion coefficients of glucose and water in the healthy and pathological mucosa tissues. When performing these calculations, we considered the corresponding thickness values obtained at a time of treatment equal to $\tau$ and for the treatment with the same glucose concentration as the one that originates a minimum (for water) and a maximum (for glucose) $\tau$ value for each tissue [45]. The results of these calculations are presented in Section 3 and they show that the maximum $\tau$ occurs for each tissue at different glucose concentrations in the treating solution. Such difference in the glucose concentration in the treating solution between the healthy and pathological mucosa indicates a different mobile water content in the two tissues, a parameter that can be used to discriminate cancer.

In the third study, $T_{c}$ spectra were also measured from healthy and pathological mucosa tissues during treatment, but now using only $93 \%$-glycerol as a treating solution [29]. Due to the fact that proteins have a strong absorption band between 200 and $230 \mathrm{~nm}[39,54]$, it was necessary to evaluate the kinetic variations created by the treatments in that deep-UV range. When $T_{c}$ spectra are represented as a function of the time of treatment, we see that the kinetics in the deep-UV range are insignificant and not perceptible when compared to the variations observed in the visible-NIR range. To get access to those kinetic variations in the deep-UV, we need to calculate the relative variations of $T_{c}$ over the time of treatment. We used Eq. (6) to perform those calculations and designated the relative variations as optical clearing efficiency $\left(O C_{\text {eff }}\right)[25,29]$ :

$$
O C_{\text {eff }}(\lambda, t)=\frac{T_{c}(\lambda, t)-T_{c}(\lambda, t=0)}{T_{c}(\lambda, t=0)} \times 100 \%,(6)
$$

where $T_{c}(\lambda, t)$ represents the $T_{c}$ spectrum of the tissue acquired at a time of treatment $t$ and $T_{c}(\lambda, t=0)$ represents the $T_{c}$ spectrum of the tissue in its native state $(t=0)$. In a previous study [25], we observed that the $O C_{\text {eff }}$ is much higher in the deep-UV range when compared to the visible-NIR range, a fact that demonstrates a more efficient RI matching and a higher scattering reduction in that range. Due to this stronger scattering reduction in the deep-UV, we were able to use the calculated $O C_{\text {eff }}$ data to evaluate the protein dissociation mechanism in healthy and pathological mucosa tissues. During this evaluation, we observed that at $200 \mathrm{~nm}$ the $O C_{\text {eff }}$ presented the highest values for both tissues. This way, we selected that wavelength to characterize the kinetics obtained for both tissues and retrieved a parameter, which we designated as the protein dissociation rate.
In a first analysis of the $O C_{\text {eff }}$ kinetics of both tissues at $200 \mathrm{~nm}$, we observed that for the treatment duration (30 $\mathrm{min}$ ) it can be characterized by a combination of an initial square root dependence with a later linear dependence on time [29]. It was also observed that for the time interval between 3 and $10 \mathrm{~min}$, both tissues present an almost perfect linear dependence between $O C_{\text {eff }}$ and time. With the objective of developing future noninvasive and fast optical methods for cancer diagnosis, we analyzed the slope of such linear fitting for both tissues between 3 and $10 \mathrm{~min}$. For the pathological mucosa the slope was approximately $3 \times$ bigger than the one obtained for the healthy mucosa. This difference indicates a higher protein dissociation rate as a result of the greater protein content in the pathological mucosa, which has been reported in literature [51]. Section 3 presents these results and the discussion for the three studies.

\section{Results and discussion}

Due to the fact that we have performed three studies that produced discriminating results, which can be used to diagnose colorectal cancer, the presentation of results and the corresponding discussion for each study will be presented in the following three sub-sections.

\subsection{Pigment as a marker of colorectal cancer}

In this first study, we measured $10 T_{t}$ and $10 R_{t}$ spectra from healthy and a similar number from pathological mucosa samples. Such measurements were made using the setups presented in Fig. 2 and in Fig. 3, respectively. Fig. 7 presents the mean and standard deviation (SD) bars of these measurements for both mucosa tissues [19].

To calculate the wavelength dependency of $\mu_{a}$ for all tissue samples used in this study, we used the individual $T_{t}$ and $R_{t}$ spectra that originated the mean data presented in Fig. 7 in Eq. (2) with the sample thickness of $0.05 \mathrm{~cm}$. The mean results of this calculation for the healthy and pathological mucosa are presented in Fig. 8 [19].

Analyzing the ratios between the hemoglobin peaks at 415 and $550 \mathrm{~nm}$ and the lowest $\mu_{a}$ value (at $875-980 \mathrm{~nm}$ ), we obtained for both tissues a 2.7 -fold at $415 \mathrm{~nm}$ and a 1.8 -fold at $550 \mathrm{~nm}$. These values are too low $(\sim 6 \times$ less $)$ when compared to the similar data that has been published for other mucosal tissues [52]. Such low ratios indicate the presence of some particular absorber that camouflages the true blood content in the colorectal mucosa tissues. Obtaining from patient's records that complementary histological analysis indicated the presence of a lipofuscin-type absorber in the mucosa tissues, we obtained the wavelength dependence of $\mu_{a}$ for lipofuscin $\left(\mu_{a-p i g m e n t}(\lambda)\right.$, see Eq. (3)) from literature [53] to subtract it to the $\mu_{a}(\lambda)$ of the healthy mucosa. Considering $A=1$ in Eq. (3) and after subtracting $\mu_{a-p i g m e n t}(\lambda)$ from $\mu_{a}(\lambda)$ of the healthy mucosa, we obtained acceptable ratios at 415 and $550 \mathrm{~nm}$ : 19.7 -fold at $415 \mathrm{~nm}$ and 10.1-fold at $550 \mathrm{~nm}$. Using the same procedure for the pathological mucosa, but now with $A=1.1$ in Eq. (3) (an additional 10\% lipofuscin 
accumulation), we obtained the hemoglobin ratios: 33.1-fold at $415 \mathrm{~nm}$ and 17.3-fold at $550 \mathrm{~nm} \mathrm{[19].}$ Analyzing these data, we see that the pathological mucosa presents higher blood content than the healthy mucosa, as expected [55]. The results obtained after subtracting lipofuscin to the $\mu_{a}(\lambda)$ of the colorectal mucosa tissues and the new hemoglobin ratios are presented in Fig. 9.

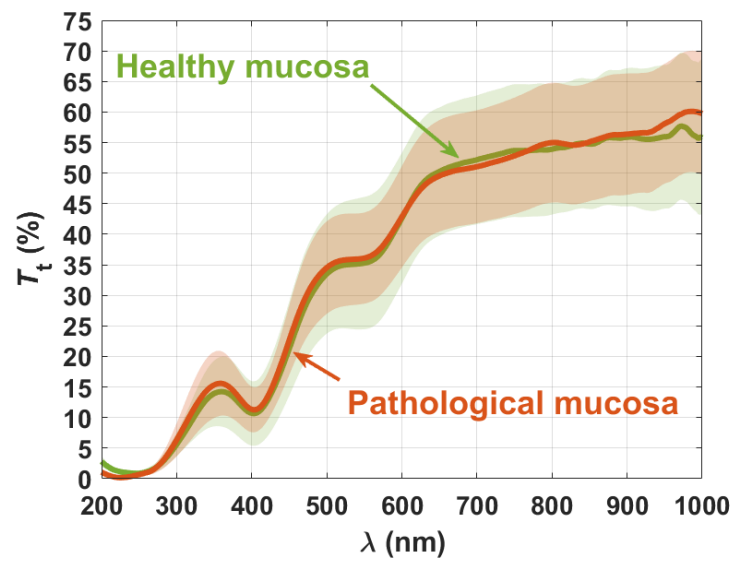

(a)

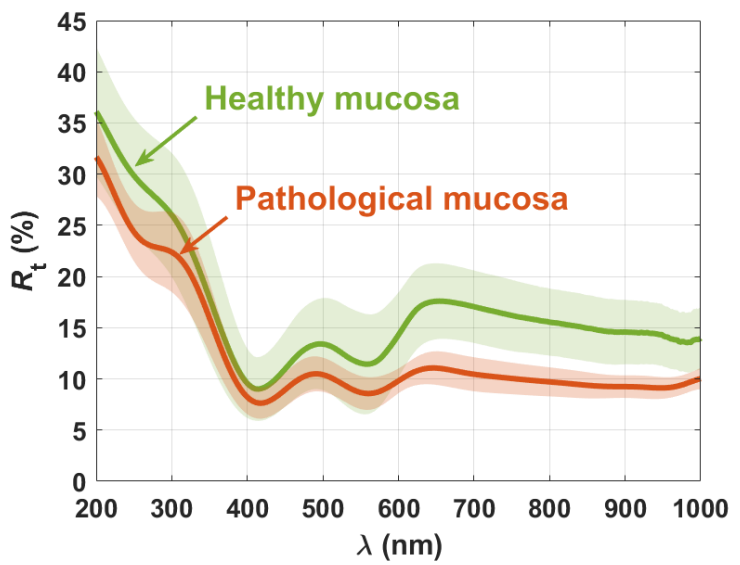

(b)

Fig. 7 Mean $T_{t}(a)$ and $R_{t}(b)$ spectra for the healthy and pathological mucosa.

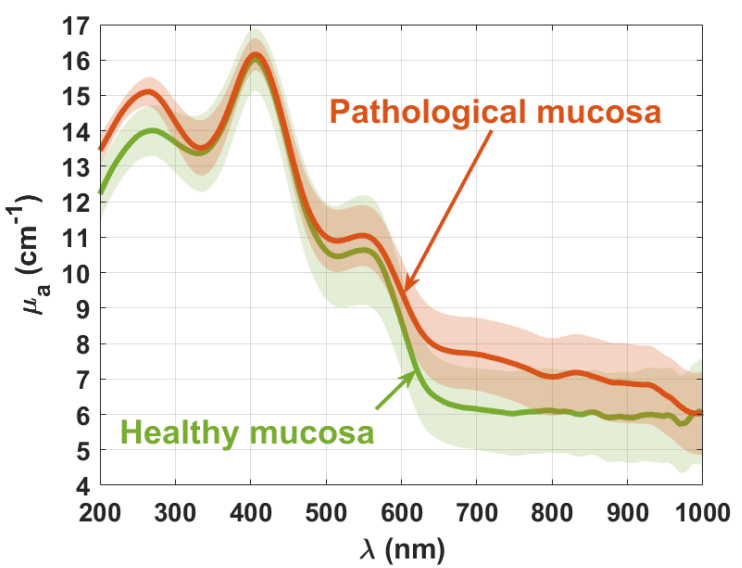

Fig. 8 Mean wavelength dependence for $\mu_{a}$ of both mucosa tissues.

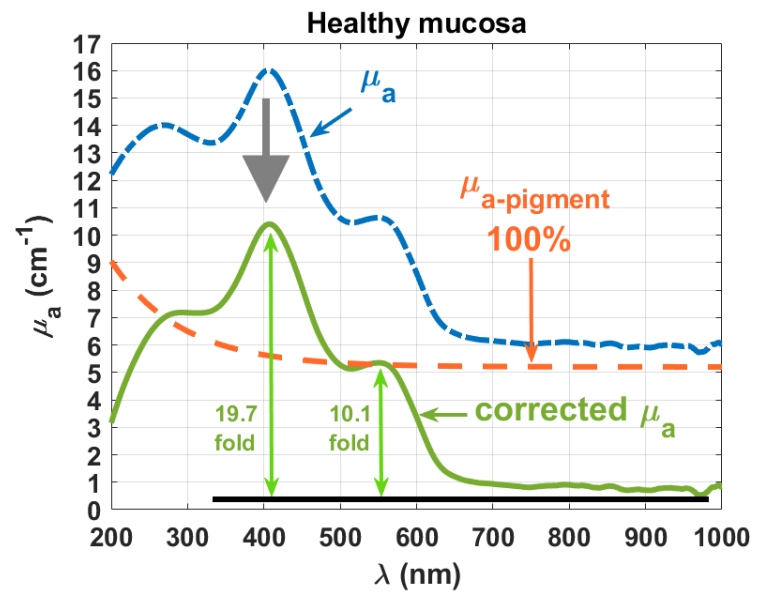

(a)

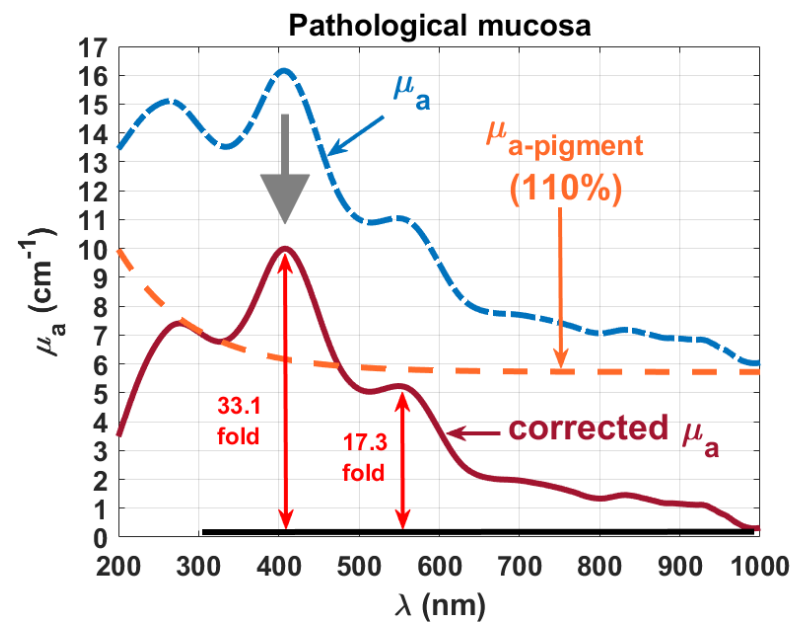

(b)

Fig. 9 Absorption coefficient $\mu_{a}(\lambda)$ for the pigment, for healthy (a) and for pathological (b) mucosa, before and after subtracting the absorption of the pigment.

Since the results obtained for the $\mu_{a}(\lambda)$ of the mucosa tissues were calculated from spectra that were acquired in an invasive manner, we wanted to check if these results could be obtained from noninvasive measurements and allow for the development of a diagnostic procedure without the need of surgical resections. Due to the fact that this research is at an early stage of development, no conditions were assembled to perform measurements in vivo from patients. Still, using the configuration presented in Fig. 4, we acquired the $R_{d}$ spectra from 10 healthy and 10 pathological mucosa samples to mimic the noninvasive in vivo measurements. This is a new set of tissue samples, that we have prepared only to test the hypothesis of reconstructing $\mu_{a}(\lambda)$ from measurements that mimic the in vivo approach. Using the $20 R_{d}$ spectra (10 from healthy and 10 from pathological mucosa) as input to various ML algorithms, we were able to select the Random Forrest Regression (RFR) algorithm as the best choice to reconstruct the $\mu_{a}(\lambda)$ of healthy and pathological mucosa tissues [20]. The resulting $\mu_{a}$ spectra, as obtained from RFR reconstruction and after 
subtracting the pigment absorption are presented in Fig. 10.

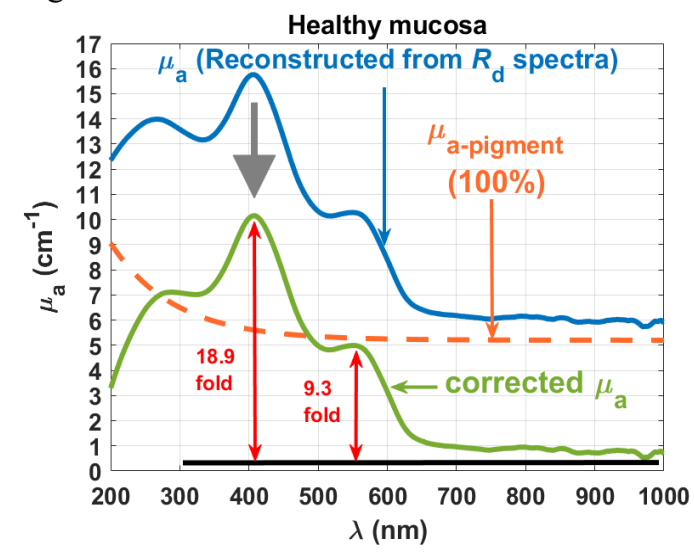

(a)

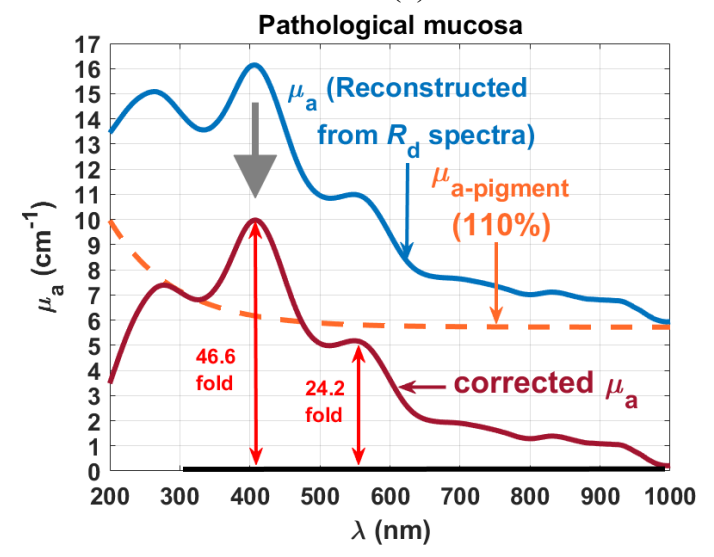

(b)

Fig. 10 Absorption coefficient $\mu_{a}(\lambda)$ for the pigment, for healthy (a) and for pathological (b) mucosa, as reconstructed by the RFR algorithm and after subtracting the absorption of the pigment.

Comparing the ratios obtained in Fig. 10 with the ones presented in Fig. 9, we see that for the healthy mucosa they have similar magnitude. For the case of the pathological mucosa, we obtained higher magnitude ratios with the ML approach, both at 415 and at $550 \mathrm{~nm}$. This means that the pathological samples used to measure the $R_{d}$ spectra that was used in the ML estimation might have a higher blood content than the ones used to obtain the original results that are presented in Fig. 8.

The results presented in Fig. 10 show that it is possible to measure broad-band $R_{d}$ spectra from tissues in vivo and use them in ML algorithms to reconstruct $\mu_{a}(\lambda)$ for those tissues. Moreover, when the diagnostic information is directly available in those absorption spectra, or can be calculated in a simple manner, as in the case presented above, the ML algorithms are a good choice for a noninvasive diagnostic approach. Such method presents an additional advantage, since it can be used to perform a diagnosis in an early stage of cancer development, provided that the pigment content is known for the different stages of cancer progression.

\subsection{Mobile water content as a marker of colorectal cancer}

In this second study, to evaluate the diffusion properties of glucose and water in the colorectal mucosa tissues, the tissue samples were treated with aqueous solutions containing different osmolarities of glucose. Three studies were performed for each glucose osmolarity to obtain mean $T_{c}$ kinetics at discrete wavelengths between 600 and $800 \mathrm{~nm}$ over the time of treatment $[43,45]$. The mean $T_{c}$ kinetics obtained for such wavelengths in some of the treatments applied to the healthy and pathological mucosa are presented in Fig. 11.

The various graphs in Fig. 11 show that when the treatment is made with low glucose osmolarity, $T_{c}$ increases until a maximum is reached after a few minutes of treatment. The time when such maximum is reached depends on the glucose concentration in the treating solution. After reaching such maximum, a smooth decrease in the $T_{c}$ kinetics is observed for the treatments with $25 \%$-glucose in graphs (a) and (b) of Fig. 11. Such smooth decrease indicates a possible water back-flux into the tissue and some tissue swelling. Such $T_{c}$ kinetics indicate that the effective glucose flux into the tissue is limited to the first few minutes of treatment, nearly 6 min for the healthy and $8 \mathrm{~min}$ for the pathological mucosa. As the glucose concentration in the treating solution increases to intermediate values, a smooth increase in $T_{c}$ is obtained during the entire treatment. Such behavior is seen in graphs (c) and (d) of Fig. 11, for the treatments of pathological mucosa with 35\%-glucose and healthy mucosa with $40 \%$-glucose. In these treatments, the smooth increase is observed for the entire treatment duration $-30 \mathrm{~min}$, without reaching a maximum before that time. Such behavior indicates a unique glucose flux into the tissue as a result of a water balance between the treating solution and the mobile water in the tissue. If we increase the glucose concentration in the treating solution to higher values, we see that the smooth increase in the $T_{c}$ kinetics becomes limited to shorter times of treatment (see graphs (e) and (f) in Fig. 11), as observed for the treatments with low glucose concentrations (graphs (a) and (b) in Fig. 11). The difference between the treatments with low and high glucose concentrations is that in the later no smooth $T_{c}$ decrease is observed at the end of the treatment. In these treatments, $T_{c}$ stays almost unchanged at the end of treatment, indicating that no effective net flux occurs.

The data in graphs of Fig. 11 and similar data obtained from the other treatments with other glucose concentrations needed to be processed to obtain the mean $\tau$ for each treatment. This way, for a particular treatment, and considering the time span where the $T_{c}$ kinetics show increasing behavior, each dataset that correspond to an individual wavelength was first displaced to have $T_{c}(t=0)=0$. The next step was to normalize the dataset to its highest value, so that it varies between 0 and 1 . Finally the rearranged dataset was fitted with a curve described by Eq. (4) to estimate the corresponding $\tau$ value. 


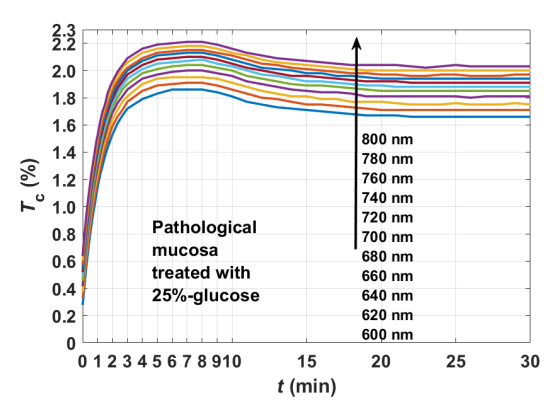

(a)

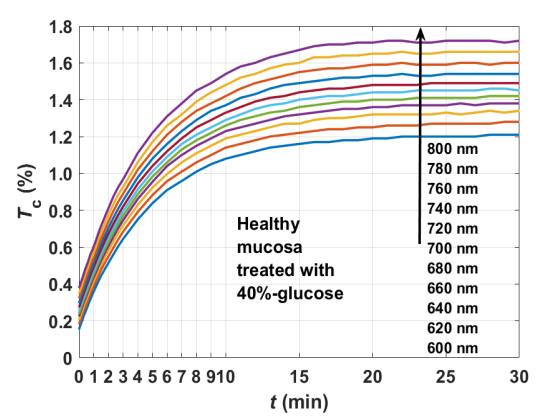

(d)

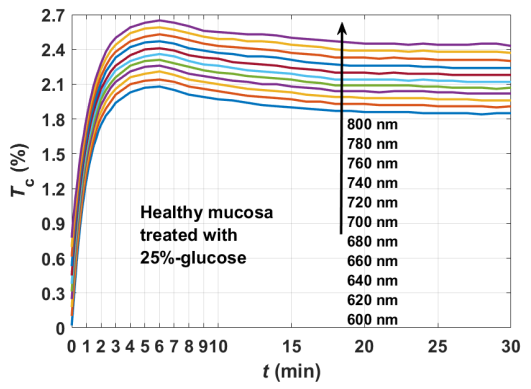

(b)

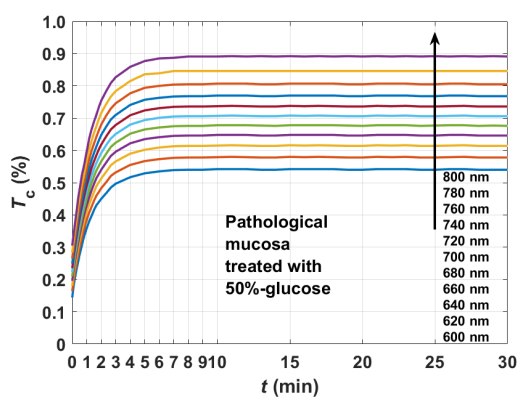

(e)

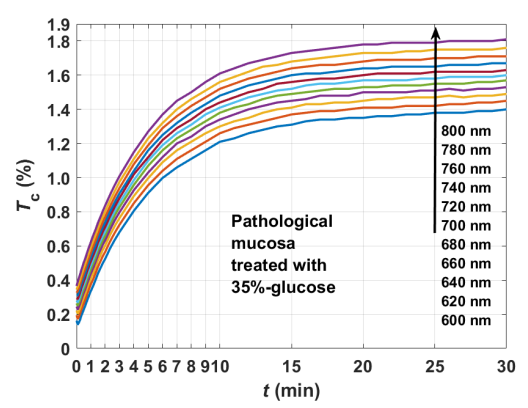

(c)

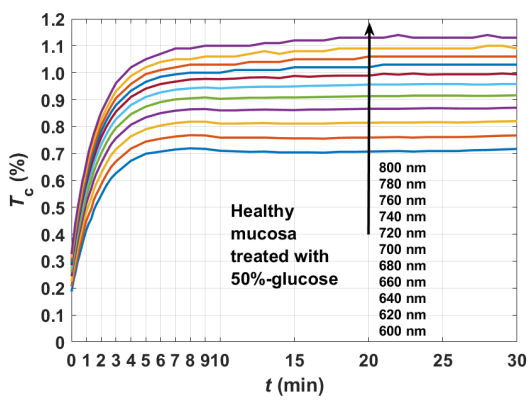

(f)

Fig. 11 Mean $T_{c}$ kinetics for the healthy and pathological mucosa under treatment with: 25\%-glucose (a and b), $35 \%$-glucose (c), 40\%-glucose (d) and 50\%-glucose (e and f).

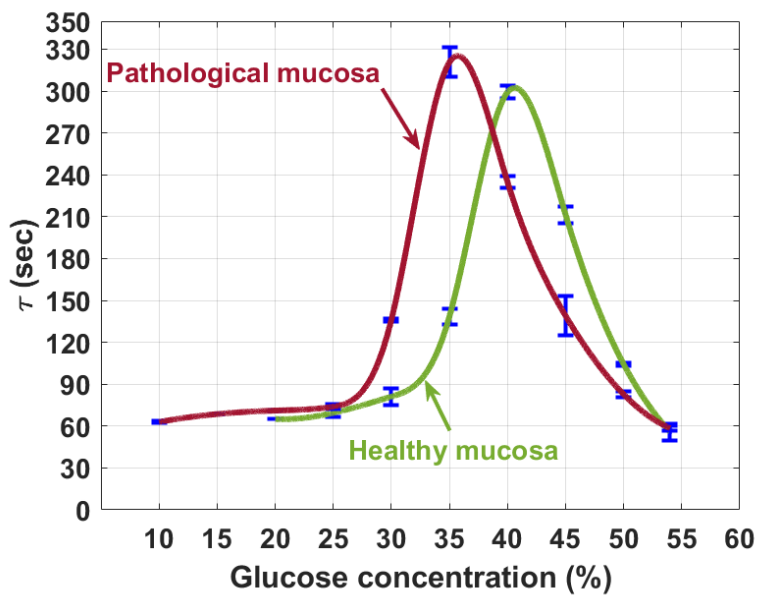

Fig. 12 Mean $\tau$ as a function of glucose concentration in the treating solution for human healthy and pathological colorectal mucosa tissues.

Since we have obtained various $\tau$ values for each treatment, the mean and SD for $\tau$ were calculated for each treatment. Fig. 12 presents such values for the treatments applied to the healthy and pathological mucosa tissues [43, 45].

Considering each tissue, the points in Fig. 12 were adjusted with a spline to obtain the dependence between the $\tau$ values that characterize the effective flux that occurs in each treatment and the glucose concentration in the treating solution. These curves show a peak at particular glucose concentrations in the treating solution. The $\tau$ values at these peaks represent the true glucose diffusion time in the healthy and pathological mucosa tissues, and the glucose concentration where they occur is indicative of the mobile water content in each case. For the healthy mucosa we see that the maximum $\tau$ is $302.4 \mathrm{sec}$ and it occurs for a glucose concentration of $40.6 \%$. This means that the mobile water content in the healthy mucosa is $59.4 \%$. For the pathological mucosa, the maximum $\tau$ is $325.1 \mathrm{~s}$ and it occurs for a glucose concentration of $35.7 \%$, meaning that the mobile water content in this tissue is $64.3 \%$. These results show that the mobile water content is almost $5 \%$ higher in the pathological mucosa $[43,45]$, which leads us to believe that during cancer progression, part of the bound water is converted into mobile. For the treatment with $54 \%$-glucose, we retrieve from Fig. 12 a minimum value for $\tau$ in both tissues: $55.7 \mathrm{sec}$ in the healthy and $58.4 \mathrm{sec}$ in the pathological mucosa.

In face of the results obtained from Fig. 12 and with the objective of calculating the diffusion coefficients of glucose and water in both tissues, we performed thickness kinetic measurements from the healthy mucosa under treatment with $40 \%$ - and $54 \%$-glucose and from the pathological mucosa under treatment with $35 \%$ - and $54 \%$-glucose. These measurements were made three times with individual samples for each treatment and were done using the setup presented in Fig. 6. The mean results of thickness kinetics obtained from these measurements are presented in Fig. 13.

To calculate the diffusion coefficients of water and glucose in the mucosa tissues and considering each 
treatment for which we performed the thickness measurements, we retrieved from the corresponding dataset in graphs of Fig. 13 the thickness value that correspond to the mean $\tau$ value obtained for that treatment. Using both the mean $\tau$ and mean thickness values, as retrieved for each treatment from graphs in Figs. 12 and 13, in Eq. (5), we calculated the diffusion coefficients of water and glucose in the healthy and pathological mucosa tissues. The results of these calculations were: $D_{\text {glucose }}=5.8 \times 10^{-7} \mathrm{~cm}^{2} / \mathrm{sec}$ and $D_{\mathrm{H} 2 \mathrm{O}}=3.3 \times 10^{-6} \mathrm{~cm}^{2} / \mathrm{sec}$ in the healthy mucosa and $D_{\text {glucose }}=4.4 \times 10^{-7} \mathrm{~cm}^{2} / \mathrm{sec}$ and $D_{\mathrm{H} 2 \mathrm{O}}=2.4 \times 10^{-6} \mathrm{~cm}^{2} / \mathrm{sec}$ in the pathological mucosa $[21,43,45]$. These values can also be used for colorectal cancer discrimination, since they differ from the healthy to the pathological mucosa.

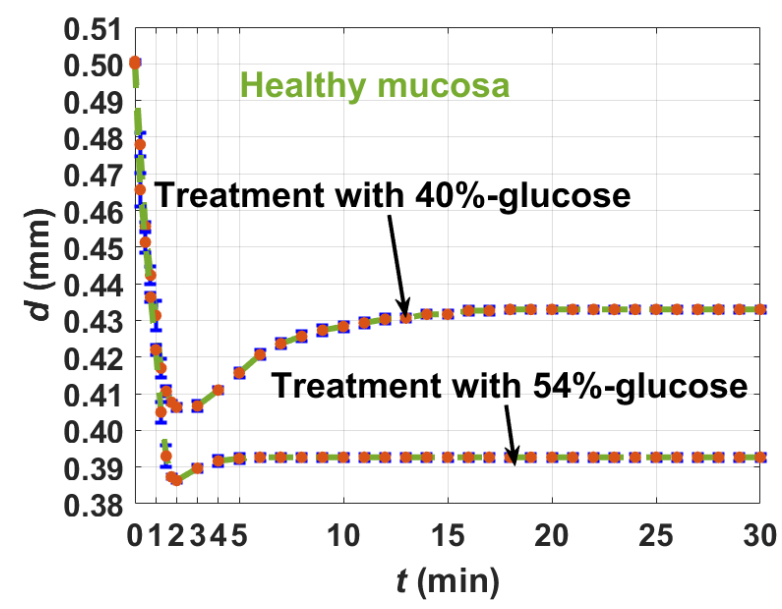

(a)

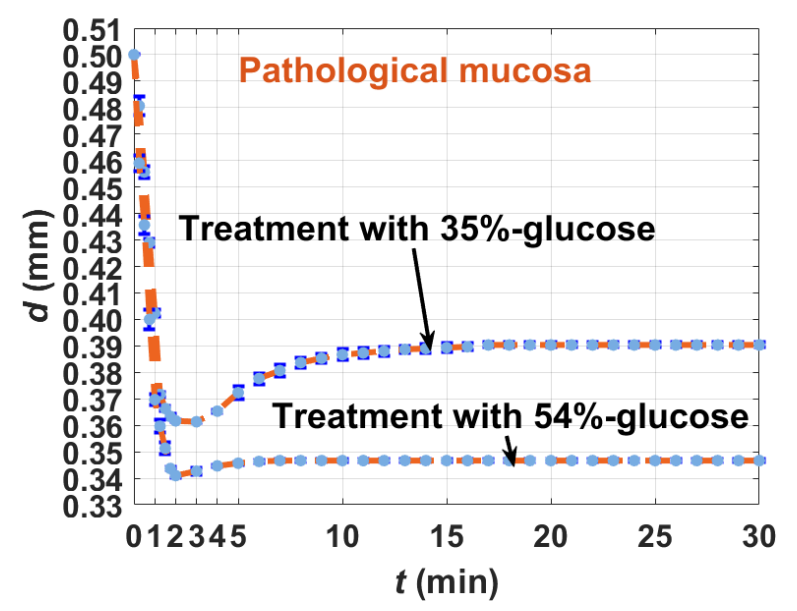

(b)

Fig. 13 Mean thickness kinetic measurements from mucosa tissues: healthy mucosa under treatment with $40 \%$ - and 54\%-glucose (a), and pathological mucosa under treatment with 35\%- and 54\%-glucose (b).

To finalize this study, it is important to say that, although we have obtained these results based on invasive measurements, they can also be obtained using in vivo noninvasive optical procedures. The estimation of the $\tau$ values from $T_{c}$ kinetics, can be done if we replace the invasive measurements of $T_{c}$ spectra by noninvasive measurements of $R_{d}$ spectra, which are also sensitive to the changes created in the tissue by the OC treatments. Regarding the thickness kinetics that are necessary to calculate the diffusion coefficients, they can also be obtained by noninvasive OCT measurements [21]. This way, combining $R_{d}$ kinetics with OCT thickness measurements obtained from in vivo tissues under treatment with OCA solutions, the diffusion properties can be evaluated, a procedure that ultimately allows the estimation of the mobile water content in the tissues, a parameter that can be used to perform a diagnosis.

\subsection{Differentiated protein dissociation rate in healthy and pathological mucosa}

In this final study, both healthy and pathological colorectal mucosa tissues were submitted to $T_{c}$ measurements during treatment with 93\%-glycerol [29]. To obtain statistical data, 5 healthy and 5 pathological samples were submitted to these treatments, and the spectra were acquired between 200 and $1000 \mathrm{~nm}$ for a treatment time of $30 \mathrm{~min}$. After terminating the measurements, the mean time dependence for the $T_{c}$ spectra was calculated for each tissue, showing neglectable variations in the deep-UV range when compared to the variations observed for the visible-NIR range [29]. Since proteins present a strong absorption band in the 200-230 $\mathrm{nm}$ range [39, 54], we wanted to get access the variations induced by the glycerol molecules in that range. To obtain significant kinetic variations in the deep-UV, we used the mean $T_{c}$ spectra in Eq. (6) to calculate the $O C_{\text {eff }}$ as a function of time and wavelength in each tissue [29]. Fig. 14 presents the results of these calculations for both tissues.

According to literature [39, 54], the major absorption band of proteins occurs in the 200-230 nm range, but from graphs in Fig. 14, we see that the highest $O C_{\text {eff }}$ is located at $200 \mathrm{~nm}$. This means that the protein dissociation mechanism in colorectal mucosa tissues is translated in a higher efficiency at this wavelength. For this reason, we selected this wavelength to perform a further analysis and try to discriminate the pathology.

To perform such further analysis, we represented the $O C_{\text {eff }}$ kinetics at $200 \mathrm{~nm}$ for both tissues in Fig. 15.

Considering the mean $O C_{\text {eff }}$ points for each tissue in Fig. 15, we see that both kinetics are well fitted by a curve that is described by a square-root of time at the beginning of the treatment and a linear dependence on time at later treatment (purple lines in Fig. 15) [29]. With the objective of developing future noninvasive diagnostic procedures that should be fast, we observed that the $O C_{\text {eff }}$ kinetics for both tissues also present a linear time dependence between 3 and $10 \mathrm{~min}$ of treatment (brown and green lines in Fig. 15). We calculated the linear fitting of the $O C_{\text {eff }}$ points for each tissue in the 3-10 min range, obtaining the following equations [29]:

$$
O C_{e f f-n m}=27.4 \times t+189.6
$$




$$
O C_{e f f-p m}=79.1 \times t+721.5,
$$

where $O C_{\text {eff-nm }}$ is the $O C_{\text {eff }}$ for the healthy mucosa and $O C_{\text {eff-pm }}$ is the $O C_{\text {eff }}$ for the pathological mucosa, both represented in $\%$ and $t$ represents the time of treatment in $\min$.

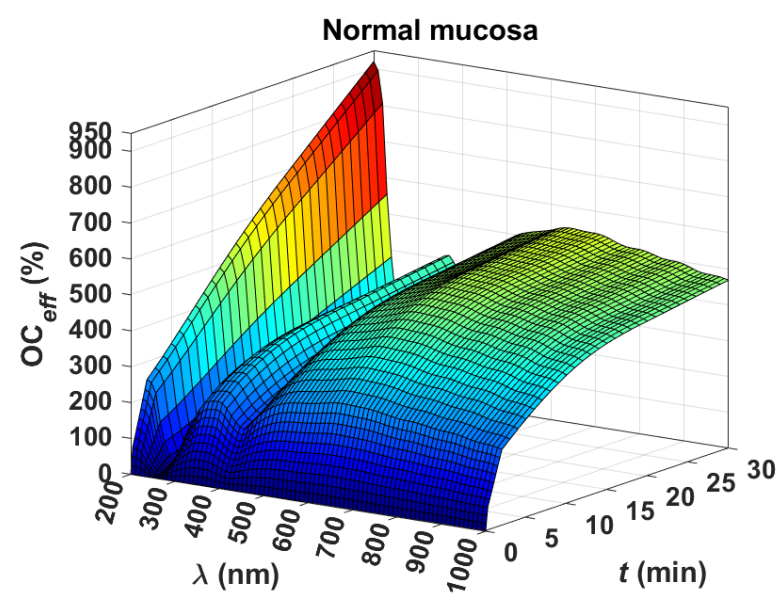

(a)

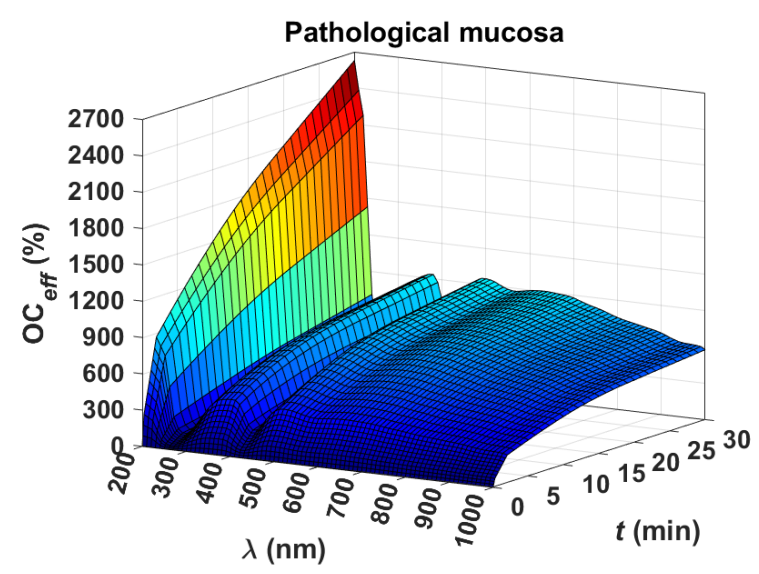

(b)

Fig. 14 Mean $O C_{\text {eff }}$ kinetics for the treatments of healthy mucosa (a) and pathological mucosa (b) with 93\%glycerol.

From the resulting equations we see that the slope obtained for the pathological mucosa is almost $3 \times$ higher than the one obtained for the healthy mucosa, meaning that the diseased tissue contains more proteins that are dissociated during the $\mathrm{OC}$ treatments. The discriminated slope of the $O C_{\text {eff }}$ kinetics can be used as a measure of the protein dissociation rate for future cancer diagnostic procedures, which based on noninvasive $R_{d}$ measurements in the deep-UV can reproduce the results obtained in this study.

Due to the known fact that proteins accumulate in a higher amount in cancer tissues [51], and such accumulation should increase with cancer progression, further studies should be made to obtain a relation between the protein dissociation rate and the stage of cancer development. Due to the different nature of the various types of cancer, such studies should be performed not only for colorectal cancer, but for other cancers as well.

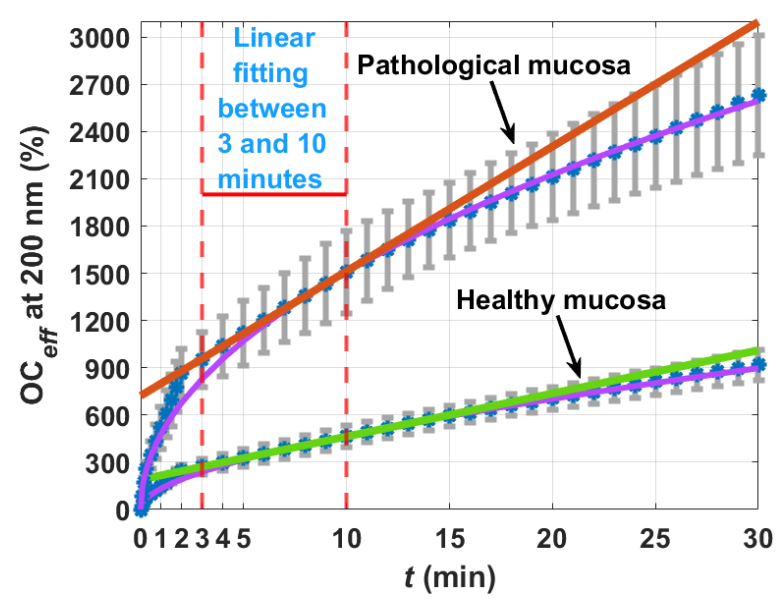

Fig. 15 Mean $O C_{\text {eff }}$ kinetics at $200 \mathrm{~nm}$ for healthy and pathological mucosa during treatment with 93\%glycerol, dual time dependence fitting for the entire treatment (purple lines) and linear fitting between 3 and $10 \mathrm{~min}$ (brown and green lines).

\section{Conclusions}

The results obtained in the above studies show that it is possible to discriminate colorectal cancer through optical spectroscopy methods. Although these results were obtained from ex vivo transmittance spectroscopy, noninvasive $R_{d}$ spectroscopy methods can be applied in the in vivo situation, since both the $T_{c}$ and $R_{d}$ configurations are sensitive to obtain the parameters and variations presented above, which can be used for cancer discrimination and monitoring.

In the first study, the simple measurements of $T_{t}$ and $R_{t}$ spectra allowed to identify a discriminated content of a pigment in healthy and pathological colorectal mucosa tissues. The detection of such pigment and the evaluation of different content in healthy and diseased tissues was made with simple calculations, using the absorption coefficient spectra of both tissues. Such spectral optical property is traditionally obtained from data that is measured with invasive procedures from ex vivo tissues, but with the application of ML techniques, we have demonstrated that it is possible to estimate it with significant precision from noninvasive $R_{d}$ measurements, which can be applied to the in vivo situation.

When performing the second and third studies, we wanted to acquire information to characterize the three OC mechanisms, but the methods used and their application both to healthy and pathological colorectal tissues has proven that diagnostic information can also be acquired during OC treatments. One important result from the second study is the different water content that was found in both tissues. Such different water content values in healthy and cancer tissues show that bound water is converted into mobile water during the progress of cancer in tissues and the evaluation of its content 
provides diagnostic information. Similar studies to investigate the water content in healthy and cancer tissues should be performed for other types of cancer and in different stages of cancer progression to establish a dependence relation and use the water content to monitor cancer progression. Although such study was based on invasive ex vivo $T_{c}$ measurements, the method and calculations involved can easily be adapted to use noninvasive and in vivo $R_{d}$ spectra to reach similar results.

In the third study, by knowing that the protein content is higher in cancer-diseased than in healthy tissues, we applied a solution with high-osmolarity glycerol to treat the tissues and study the OC mechanism of protein dissociation. Since proteins present their strong absorption in the deep UV, a spectral range where scattering is also strong, tissues present the lowest transmittance in that range. When $\mathrm{OC}$ treatments are applied, low-magnitude variations are observed in the $T_{c}$ kinetics, a fact that does not allow for direct evaluation of the protein dissociation mechanism. To overcome such difficulty and evaluate the protein dissociation rates in healthy and pathological mucosa tissues, we calculated the OC efficiency as the relative variations of $T_{c}$ during treatment. Analyzing such calculated data, we obtained a higher rate for protein dissociation in the pathological tissue ( $\sim 3 \times$ higher), a result that is consistent with the fact of higher protein content in cancer tissues. Once again such discriminating data may be useful for cancer diagnosis, and it can also be obtained from in vivo $R_{d}$ measurements. Nevertheless, further studies should be made with other types of cancer and in different stages of their progression to quantify the protein content in tissue from different stages of cancer progression. Only by obtaining such information, it will be possible to develop a clinical in vivo procedure to diagnose cancer and monitor its progression.

\section{Disclosures}

All authors declare that there is no conflict of interests in this paper.

\section{Acknowledgements}

The authors of the article knew well and communicated with Ekaterina Borisova for many years, especially Valery Tuchin and in recent years Luís Oliveira. We had many joint plans to organize conferences and joint research projects, but COVID-19 mercilessly interrupted our communication. We have lost a great scientist and a person with a huge soul, sociable, but at the same time modest and kind. We will always remember our warm meetings and fruitful work with Ekaterina.

This research was supported by the Portuguese grant FCT-UIDB/04730/2020.

VVT was supported by grant under the Decree of the Government of the Russian Federation No. 220 of 09 April 2010 (Agreement No. 075-15-2021-615 of 04 June 2021).

\section{References}

1. V. V. Tuchin, L. M. Oliveira, "Recent progress in tissue enhanced spectroscopy for cancer detection," Asian Journal of Physics 30(3), 427-444 (2021).

2. J. Vignard, G. Mirey, and B. Salles, "Ionizing-radiation induced DNA double-strand breaks. A direct and indirect lighting up," Radiotherapy and Oncology 108(3), 362-369 (2013).

3. G. Borrego-Soto, R. Ortiz-López, and A. Rojas-Martinez, "Ionizing radiation-induced DNA injury and damage detection in patients with breast cancer," Genetics and Molecular Biology 38(4), 420-432 (2015).

4. J.-M. Cosset, "Side effects of ionizing radiation on healthy tissues and organs at risk," Cancer Radiotherapie : Journal de la Societe Francaise de Radiotherapie Oncologique 14(4-5), 228-231 (2010).

5. O. Popanda, J. W. Marquardt, J. Chang-Claude, and P. Schmezer, "Genetic variation in normal tissue toxicity induced by ionizing radiation," Mutation Research/Fundamental and Molecular Mechanisms of Mutagenesis 667(1-2), 5869 (2009).

6. T. N. Seyfried, R. E. Flores, A. M. Poff, and D. P. D’Agostino, “Cancer as a metabolic disease: implications for novel therapeutics," Carcinogenesis 35(3), 515-527 (2014).

7. M. B. Sporn, "The war on cancer," The Lancet 347(9012), 1377-1381 (1996).

8. A. V. Lichtenstein, "Strategies of the War on Cancer: To kill or to Neutralize?" Frontiers in Oncology 8, 667 (2018).

9. T. N. Seyfried, Cancer As a Metabolic Disease: On the Origin, Management, and Prevention of Cancer, John Wiley \& Sons, Hoboken (NJ), USA (2012). ISBN: 978-0-470-58492-7.

10. I. J. Fidler, "The pathogenesis of cancer metastasis: the 'seed and soil' hypothesis revisited," Nature Reviews Cancer 3(6), 453-458 (2003).

11. G. P. Gupta, J. Massagué, “Cancer metastasis: building a framework,” Cell 127(4), 679-695 (2006).

12. Y. Lazebnik, "What are the hallmarks of cancer?" Nature Reviews Cancer 10, 232-233 (2010).

13. D. Tarin, "Cell and tissue interactions in carcinogenesis and metastasis and their clinical significance," Seminars Cancer Biology 21(2), 72-82 (2011). 
14. D. M. Parkin, J. Stjernswärd, and C. S. Muir, "Estimates of the worldwide frequency of twelve major cancers," Bulletin of the World Health Organization 62(2), 163-182 (1984).

15. V. V. Tuchin, J. Popp, and V. Zakharov (Eds.), Multimodal Optical Diagnostics of Cancer, Springer Nature, Switzerland (2020). ISBN: 978-3-030-44593-5.

16. J. Ferlay, I. Soerjomataram, R. Dikshit, S. Eser, C. Mathers, M. Rebelo, D. M. Parkin, D. Forman, and F. Bray, "Cancer incidence and mortality worldwide:sources, methods and major patterns in GLOBOCAN 2012," International Journal of Cancer 136(5), E359-E386 (2015).

17. F. Bray, J. Ferlay, I. Soerjomataram, R. L. Siegel, L. A. Torre, and A. Jemal, "Global cancer statistics 2018: GLOBOCAN estimates of incidence and mortality worldwide for 36 cancers in 185 countries," CA: A Cancer Journal for Clinicians 68(6), 394-424 (2018).

18. E. A. Genina, L. M. C. Oliveira, A. N. Bashkatov, and V. V. Tuchin, "Optical Clearing of Biological Tissues: Prospects of Application for Multimodal Malignancy Diagnostics," in Multimodal Optical Diagnostics of Cancer, V. V. Tuchin, J. Popp, V. Zakharov (eds.), Springer, Cham, Switzerland, 107-131 (2020).

19. S. Carvalho, I. Carneiro, R. Henrique, V. Tuchin, and L. Oliveira, "Lipofuscin-type pigment as a marker of colorectal cancer," Electronics 9(11), 1805 (2020).

20. L. Fernandes, S. Carvalho, I. Carneiro, R. Henrique, V. V. Tuchin, H. P. Oliveira, and L. M. Oliveira, "Diffuse reflectance and machine learning to differentiate colorectal cancer," Chaos: An Interdisciplinary Journal of Nonlinear Science 31, 053118 (2021).

21. L. M. C. Oliveira, V. V. Tuchin, The Optical Clearing Method: A New Tool for Clinical Practice and Biomedical Engineering, Springer, Cham, Switzerland (2019). ISBN: 978-3-030-33055-2.

22. Y. Zhou, J. Yao, and L. V. Wang, “Tutorial on photoacoustic tomography,” Journal of Biomedical Optics 21, 061007 (2016).

23. V. V. Tuchin, Tissue Optics: Light Scattering Methods and Instruments for Medical Diagnosis, 3rd ed., SPIE Press, Bellingham (WA), USA (2015). ISBN: 978-1-628-41517-9.

24. V. V. Tuchin, Optical Clearing of Tissues and Blood, SPIE Press, Bellingham (WA), USA (2005). ISBN: 978-0819-48110-8.

25. I. Carneiro, S. Carvalho, R. Henrique, L. Oliveira, and V. Tuchin, "Moving tissue spectral window to the deepultraviolet via optical clearing," Journal of Biophotonics 12(12), e201900181 (2019).

26. W. Spalteholz, Über das Durchsichtigmachen von menschlichen und tierischen Präparaten und seine theoretischen Bedingungen: Nebst Anhang: Über Knochenfärbung, Leipzig Hirzel, Germany (1911).

27. W. Spalteholz, Über das Durchsichtigmachen von menschlichen und tierischen Präparaten und seine theoretischen Bedingungen: Nebst Anhang: Über Knochenfärbung, Leipzig Hirzel, Germany (1914).

28. N. Gomes, V. V. Tuchin, and L. M. Oliveira, "Refractive index matching efficiency in colorectal mucosa treated with glycerol," IEEE Journal of Selected Topics in Quantum Electronics 27(4), 7200808 (2021).

29. I. Carneiro, S. Carvalho, R. Henrique, A. Selifonov, L. Oliveira, and V. V. Tuchin, "Enhanced ultravioleta spectroscopy by optical clearing for biomedical applications," IEEE Journal of Selected Topics in Quantum Electronics 27, 7200108 (2021).

30. L. Oliveira, M. I. Carvalho, E. Nogueira, and V. V. Tuchin, "Skeletal muscle dispersion (400-1000 nm) and its kinetics at optical clearing," Journal of Biophotonics 11(1), e20170094 (2018).

31. L. Oliveira, A. Lage, M. Pais Clemente, and V. V. Tuchin, "Rat muscle opacity decrease due to the osmosis of a simple mixture," Journal of Biomedical Optics 15(5), 0055004 (2010).

32. L. Oliveira, Study of the spectral transmission response of biological tissues under the influence of different osmotic agents, FEUP Edições, Porto, Portugal (2007).

33. L. Oliveira, A. Lage, M. Pais Clemente, and V. V. Tuchin, "Optical characterization and composition of abdominal Wall muscle from rat," Optics and Lasers in Engineering 47, 667-672 (2009).

34. X. Wen, Z. Mao, Z. Han, V. V. Tuchin, and D. Zhu, "In vivo skin optical clearing by glycerol solutions: mechanism," Journal of Biophotonics 3, 44-52 (2010).

35. D. Zhu, K. V. Larin, Q. Luo, and V. V. Tuchin "Recent progress in tissue optical clearing," Laser \& Photonics Reviews 7(5), 732-757 (2013).

36. D. K. Tuchina, I. G. Meerovich, O. Sindeeva, V. V. Zherdeva, A. P. Savitsky, A. A. Bogdanov Jr, and V. V. Tuchin, "Magnetic resonance contrast agents in optical clearing: prospects for multimodal tissue imaging," Journal of Biophotonics 13, e20190249 (2020).

37. A. Y. Sdobnov, M. E. Darvin, J. Schleusener, J. Lademann, and V. V. Tuchin, "Hydrogen bound water profiles in the skin influenced by optical clearing molecular agents - quantitative analysis using confocal Raman microscopy," Journal of Biophotonics 12, e201800283 (2019).

38. C. S. Choe, J. Lademann, and M. E. Darvin, "Depth profiles of hydrogen bound water molecule types and their relation to lipid protein interaction in the human stratum corneum in vivo," Analyst 141, 6329-6337 (2016).

39. T. Yu, D. Zhu, L. Oliveira, E. Genina, A. Bashkatov, and V. V. Tuchin, "Tissue optical clearing mechanisms" in Tissue Optical Clearing: New Prospects in Optical Imaging, D. Zhu, E. Genina, V. Tuchin (Eds.), CRC Press, Boca Raton, USA (2021). 
40. L. M. Oliveira, M. I. Carvalho, E. Nogueira, and V. V. Tuchin, "Diffusion characteristics of ethylene glycol in skeletal muscle," Journal of Biomedical Optics 20, 051019 (2015).

41. L. Oliveira, M. I. Carvalho, E. Nogueira, and V. V. Tuchin, "Optical clearing mechanisms characterization in muscle,” Journal of Innovative Optical Health Sciences 9(5), 1650035 (2016).

42. L. M. Oliveira, M. I. Carvalho, E. Nogueira, and V. V. Tuchin, "The characteristic time of glucose diffusion measured for muscle tissue at optical clearing," Laser Physics 23, 075606 (2009).

43. I. Carneiro, S. Carvalho, R. Henrique, L. Oliveira, and V. V. Tuchin, "A robust ex vivo method to evaluate the Diffusion properties of agents in biological tissues," Journal of Biophotonics 12, e201800333 (2019).

44. R. Cicchi, D. Sampson, D. Massi, and F. S. Pavone, "Contrast and depth enhancement in two-photon microscopy of human skin ex vivo by use of optical clearing agents," Optics Express 13(7), 2337-2344 (2005).

45. S. Carvalho, N. Gueiral, E. Nogueira, R. Henrique, L. Oliveira, and V. V. Tuchin, "Glucose Diffusion in colorectal mucosa - a comparative study between normal and cancer tissues," Journal of Biomedical Optics 22(9), 091506 (2017).

46. D. K. Tuchina, A. N. Bashkatov, A. B. Bucharskaya, E. A. Genina, and V. V. Tuchin, "Study of glycerol diffusion in skin and myocardium ex vivo under the conditions of developing alloxan-induced diabetes," Journal of Biomedical Photonics \& Engineering 3(2), 020302 (2017).

47. A. Yeh, B. Choi, J. S. Nelson, and B. J. Tromberg, "Reversible dissociation of collagen in tissues," Journal of Investigative Dermatology 121(6), 1332-1335 (2003).

48. J. Hirshburg, B. Choi, J. S. Nelson, and A. T. Yeh, "Collagen solubility correlates with skin optical clearing,” Journal of Biomedical Optics 11(4), 040501 (2006).

49. J. Hirshburg, B. Choi, J. S. Nelson, and A. T. Yeh, “Correlation between collagen solubility and skin optical clearing using sugars," Lasers in Surgery and Medicine 39(2), 140-144 (2007).

50. J. Hirshburg, K. M. Ravikumar, W. Hwang, and A. T. Yeh, "Molecular basis for optical clearing of collagenous tissues," Journal of Biomedical Optics 15(5), 055002 (2010).

51. S. Peña-Llópis, J. Brugarolas, "Simultaneous isolation of high-quality DNA, RNA, miRNA and proteins from tissues for genomic applications," Nature Protocols 8, 2240-2255 (2013).

52. A. N. Bashkatov, E. A. Genina, V. I. Kochubey, V. V. Tuchin, E. E. Chikina, A. B. Knyazev, and O. V. Mareev, "Optical properties of mucous membrane in the spectral range 350-2000 nm," Optics ans Spectroscopy 97, 10431048 (2004).

53. J. D. Johansson, K. Wårdell, "Intracerebral quantitative chromophore estimation from reflectance spectra captured during deep brain stimulation implantation," Journal of Biophotonics 6(5), 435-445 (2013).

54. P. Brescia, "Micro-volume purity assessment of nuclei acids using A260/A280 ratio and structural scanning protein and nucleic acid quantification," (accessed July 2021). [https:/www.biotek.com/resources/application-notes/microvolume-purity-assessment-of-nucleic-acids-using-asub260/sub/asub280/sub-ratio-and-spectral-scanning/].

55. S. Carvalho, N. Gueiral, E. Nogueira, R. Henrique, L. Oliveira, and V. V. Tuchin, "Comparative study of the optical properties of colon mucosa and colon precancerous polyps between 400 and 1000 nm," SPIE Proceedings 10063, 100631L (2017). 